M. Ben Ayed · K. El Mehdi · M. Ould Ahmedou · F. Pacella

\title{
Energy and Morse index of solutions of Yamabe type problems on thin annuli
}

Received October 18, 2004

Abstract. We consider the Yamabe type family of problems $\left(P_{\varepsilon}\right):-\Delta u_{\varepsilon}=u_{\varepsilon}^{(n+2) /(n-2)}, u_{\varepsilon}>0$ in $A_{\varepsilon}, u_{\varepsilon}=0$ on $\partial A_{\varepsilon}$, where $A_{\varepsilon}$ is an annulus-shaped domain of $\mathbb{R}^{n}, n \geq 3$, which becomes thinner as $\varepsilon \rightarrow 0$. We show that for every solution $u_{\varepsilon}$, the energy $\int_{A_{\varepsilon}}\left|\nabla u_{\varepsilon}\right|^{2}$ as well as the Morse index tend to infinity as $\varepsilon \rightarrow 0$. This is proved through a fine blow up analysis of appropriate scalings of solutions whose limiting profiles are regular, as well as of singular solutions of some elliptic problem on $\mathbb{R}^{n}$, a half-space or an infinite strip. Our argument also involves a Liouville type theorem for regular solutions on an infinite strip.

Keywords. Elliptic PDE, critical Sobolev exponent, blow up analysis, Liouville type theorem

\section{Introduction}

In this paper we consider the following Yamabe type family of problems:

$$
\left(P_{\varepsilon}\right) \begin{cases}-\Delta u_{\varepsilon}=u_{\varepsilon}^{(n+2) /(n-2)} & \text { in } A_{\varepsilon}, \\ u_{\varepsilon}>0 & \text { in } A_{\varepsilon}, \\ u_{\varepsilon}=0 & \text { on } \partial A_{\varepsilon}\end{cases}
$$

where $A_{\varepsilon}$ is an annulus-shaped open domain in $\mathbb{R}^{n}, n \geq 3$, and $\varepsilon$ is a small positive parameter. The domain $A_{\varepsilon}$ becomes thinner as $\varepsilon \rightarrow 0$ (see the precise definition of $A_{\varepsilon}$ below).

F. Pacella was supported by MURST, Project "Metodi variazionali ed equazioni differenziali non lineari”.

Mohamed Ben Ayed: Département de Mathématiques, Faculté des Sciences de Sfax, Route Soukra, Sfax, Tunisia; e-mail: Mohamed.Benayed@fss.rnu.tn

Khalil El Mehdi: Faculté des Sciences et Techniques, Université de Nouakchott, Nouakchott, Mauritania and The Abdus Salam ICTP, Trieste, Italy; e-mail: khalil@univ-nkc.mr

Mohameden Ould Ahmedou: Eberhard-Karls-Universität Tübingen, Mathematisches Institut, Auf der Morgenstelle 10, D-72076 Tübingen, Germany; e-mail: ahmedou@everest.mathematik.uni-tuebingen.de

Filomena Pacella: Dipartimento di Matematica, Università di Roma "La Sapienza",

P.le A. Moro 2, 00185 Roma, Italy; e-mail: pacella@mat.uniroma1.it

Mathematics Subject Classification (2000): 35J65, 58E05 
We define on $H_{0}^{1}\left(A_{\varepsilon}\right)$ the functional

$$
J_{\varepsilon}(u)=\frac{1}{2} \int_{A_{\varepsilon}}|\nabla u|^{2}-\frac{n-2}{2 n} \int_{A_{\varepsilon}}|u|^{2 n /(n-2)}
$$

whose positive critical points are solutions of $\left(P_{\varepsilon}\right)$.

We denote by $m\left(u_{\varepsilon}\right)$ the Morse index of $u_{\varepsilon}$ as a critical point of the functional $J_{\varepsilon}$, that is, the number of negative eigenvalues of the linearized operator $-\Delta-\frac{n+2}{n-2} u_{\varepsilon}^{4 /(n-2)}$ in $H_{0}^{1}\left(A_{\varepsilon}\right) \cap H^{2}\left(A_{\varepsilon}\right)$.

We are mainly concerned with what happens to the energy and the Morse index of $u_{\varepsilon}$ as $\varepsilon$ tends to zero. Our main motivation for investigating such behavior of the solutions comes from the fact that information about the energy and/or spectral properties is closely related to the existence and multiplicity of solutions of nonlinear equations having variational structure. It is also related to the geometric properties of solutions to PDE problems. For details see works of Bahri [2], Bahri-Lions [4], De Figueiredo-Yang [10], Lazer-Solimini [16], Pacella [18], Ramos-Terracini-Troestler [19], Solimini [24] and Yang [26], [27].

In [5], Bahri and Lions have shown that given a sequence of solutions of some superlinear and subcritical elliptic equations with Dirichlet boundary conditions in a fixed smooth and bounded domain of $\mathbb{R}^{n}$, we have $m\left(u_{k}\right) \rightarrow+\infty$ if and only if $\left\|u_{k}\right\|_{L^{\infty}} \rightarrow$ $+\infty$, provided that the nonlinearity has a prescribed behavior at infinity. Later HarrabiRebhi-Selmi [14], Yang [27], and Aubin-Bahri [1] extended this result to more general subcritical nonlinearities.

In the critical case, Bénichou and Pomet [8] proved that for radial solutions on standard thin annuli, the energy and the Morse index tend to infinity. Our goal in this paper is to prove that this result holds true for all solutions, and also on nonstandard annuli.

To be more precise, we need to introduce some notations. Let $f$ be any smooth function

$$
f: \mathbb{R}^{n-1} \rightarrow[1,2], \quad\left(\theta_{1}, \ldots, \theta_{n-1}\right) \mapsto f\left(\theta_{1}, \ldots, \theta_{n-1}\right) .
$$

which is periodic of period $\pi$ with respect to $\theta_{1}, \ldots, \theta_{n-2}$ and of period $2 \pi$ with respect to $\theta_{n-1}$. We set

$$
S_{1}(f)=\left\{x \in \mathbb{R}^{n}: r=f\left(\theta_{1}, \ldots, \theta_{n-1}\right)\right\},
$$

where $\left(r, \theta_{1}, \ldots, \theta_{n-1}\right)$ are the polar coordinates of $x$.

For $\varepsilon$ positive small enough, we introduce the map

$$
g_{\varepsilon}: S_{1}(f) \rightarrow g_{\varepsilon}\left(S_{1}(f)\right)=S_{2}(f), \quad x \mapsto g_{\varepsilon}(x)=x+\varepsilon n_{x},
$$

where $n_{x}$ is the outward normal to $S_{1}(f)$ at $x$. We denote by $\left(A_{\varepsilon}\right)_{\varepsilon>0}$ the family of annulus-shaped open sets in $\mathbb{R}^{n}$ such that $\partial A_{\varepsilon}=S_{1}(f) \cup S_{2}(f)$.

Our main result is the following.

Theorem 1. Let $u_{\varepsilon}$ be any solution of $\left(P_{\varepsilon}\right)$. Then, as $\varepsilon \rightarrow 0$,

(i) $\int_{A_{\varepsilon}}\left|\nabla u_{\varepsilon}\right|^{2} \rightarrow+\infty$,

(ii) $m\left(u_{\varepsilon}\right) \rightarrow+\infty$

where $m\left(u_{\varepsilon}\right)$ is the Morse index of $u_{\varepsilon}$ as a critical point of the functional $J_{\varepsilon}$ defined by (1.1). 
Remark 2. Statement (i) of Theorem 1 has already been proved in [6] and [7], using completely different arguments. However, our argument proves the two statements at the same time displaying a deep connection between the energy and the spectral properties of the solutions.

During the process of proving Theorem 1 we perform some blow ups and find limit equations on $\mathbb{R}^{n}$ or a half-space or an infinite strip, and it turns out that the following Liouville type theorem that we prove in Section 4 is useful.

Theorem 3. Let $u \in C^{2}(\Omega)$ be a positive bounded solution of

$$
\text { (I) } \begin{cases}-\Delta u=u^{(n+2) /(n-2)} & \text { in } \Omega, \\ u=0 & \text { on } \partial \Omega, \\ m(u)<\infty, & \end{cases}
$$

where $m(u)$ is the number of negative eigenvalues of $-\Delta-\frac{n+2}{n-2} u^{4 /(n-2)}$ in $H_{0}^{1}(\Omega) \cap$ $H^{2}(\Omega)$ and $\Omega$ is the strip defined by

$$
\Omega=\left\{\left(x^{\prime}, x_{n}\right) \in \mathbb{R}^{n-1} \times \mathbb{R}: a<x_{n}<b\right\}, \quad a, b \in \mathbb{R} .
$$

Then $u \equiv 0$ in $\Omega$.

Our proof, which is by contradiction, relies on a careful analysis of successive scalings of solutions. Such scalings give rise to singular solutions of limiting equations as well as regular ones. The analysis of the regular solutions is based on the above Liouville type theorem, while the analysis of the singular case uses in a crucial way the blow up analysis introduced by R. Schoen, and studied extensively by Y.Y. Li. In particular, the "isolated simple" property of the blow up points in the Yamabe equation on locally conformally flat manifolds is a cornerstone in our analysis, together with the extensive use of the Pohozaev identity. However, our analysis exhibits new features which are not present in the above mentioned works. A basic difference is that in contrast to those papers, our domain changes, and a big source of worry is that it may become degenerate during the blow up process. Therefore our first aim is to scale in such a way that the limit domain does not degenerate.

Another main ingredient of the proof of Theorem 1 is to show that if the Morse index of the solutions is a positive integer $m$ then also the number of blow up points of the solutions remains bounded by $m$. This is similar to what happens in other asymptotical critical problems described by El Mehdi-Pacella [11].

The organization of the remainder of the paper is as follows. In Section 2 we start our blow up scheme, blowing up first at the global maximum of $u_{\varepsilon}$, then finding another point which is the first to escape under appropriate scaling, and conclude that each of them contributes to the total energy at least a fixed amount. Section 3, devoted to the proof of Theorem 1, shows that the process started in Section 2 does not stop after finitely many steps, and that each point contributes at least 1 to the total index of $u_{\varepsilon}$, proving that both the energy and the Morse index must be infinite. In Section 4 we prove Theorem 3, while in the Appendix we recall some well known facts about the blow up analysis of Yamabe type equations. 


\section{The blow up process}

To prove Theorem 1 , we argue by contradiction, that is, we suppose that $\left(P_{\varepsilon}\right)$ has a solution $u_{\varepsilon}$ which satisfies

$$
\left(H_{1}\right) \quad \int_{A_{\varepsilon}}\left|\nabla u_{\varepsilon}\right|^{2} \leq C_{1} \quad \text { or } \quad\left(H_{2}\right) \quad m\left(u_{\varepsilon}\right) \leq C_{2},
$$

where $C_{1}$ and $C_{2}$ are given positive constants independent of $\varepsilon$. We first recall the following result:

Lemma 4 ([6]). We have:

1. $\int_{A_{\varepsilon}}\left|\nabla u_{\varepsilon}\right|^{2} \nrightarrow 0$ as $\varepsilon \rightarrow 0$.

2. $M_{1, \varepsilon} \rightarrow+\infty$ as $\varepsilon \rightarrow 0$, where $M_{1, \varepsilon}=\left\|u_{\varepsilon}\right\|_{L^{\infty}\left(A_{\varepsilon}\right)}$.

3. There exists $c>0$ such that for $\varepsilon$ small enough, we have $\varepsilon M_{1, \varepsilon}^{2 /(n-2)} \geq c$.

Now let $A_{1, \varepsilon}=M_{1, \varepsilon}^{2 /(n-2)}\left(A_{\varepsilon}-a_{1, \varepsilon}\right)$, where $a_{1, \varepsilon} \in A_{\varepsilon}$ is such that $M_{1, \varepsilon}=u_{\varepsilon}\left(a_{1, \varepsilon}\right)$, and denote by $v_{\varepsilon}$ the function defined on $A_{1, \varepsilon}$ by

$$
v_{\varepsilon}(X)=M_{1, \varepsilon}^{-1} u_{\varepsilon}\left(a_{1, \varepsilon}+M_{1, \varepsilon}^{-2 /(n-2)} X\right) .
$$

It is easy to see that $v_{\varepsilon}$ satisfies

$$
\begin{cases}-\Delta v_{\varepsilon}=v_{\varepsilon}^{(n+2) /(n-2)}, \quad 0<v_{\varepsilon} \leq 1 & \text { in } A_{1, \varepsilon}, \\ v_{\varepsilon}(0)=1, \quad v_{\varepsilon}=0 & \text { on } \partial A_{1, \varepsilon} .\end{cases}
$$

Liouville type theorems and the Pohozaev identity on the limit domain yield

Lemma 5. We have

$$
M_{1, \varepsilon}^{2 /(n-2)} d\left(a_{1, \varepsilon}, \partial A_{\varepsilon}\right) \rightarrow+\infty \quad \text { as } \varepsilon \rightarrow 0,
$$

where $d\left(a_{1, \varepsilon}, \partial A_{\varepsilon}\right)$ denotes the distance of $a_{1, \varepsilon}$ to the boundary of $A_{\varepsilon}$.

Proof. Let $l=\lim _{\varepsilon \rightarrow 0} M_{1, \varepsilon}^{2 /(n-2)} d\left(a_{1, \varepsilon}, \partial A_{\varepsilon}\right)$. According to the proof of Lemma 2.3 of [6], we have $l>0$. Arguing by contradiction, we suppose that $l<\infty$. Then it follows from (2.2) and standard elliptic theory that there exists some positive function $v$ such that (after passing to a subsequence), $v_{\varepsilon} \rightarrow v$ in $C_{\text {loc }}^{1}(\Omega)$, where $\Omega$ is a half-space or a strip in $\mathbb{R}^{n}$, and $v$ satisfies

$$
\begin{cases}-\Delta v=v^{(n+2) /(n-2)}, \quad 0<v \leq 1 & \text { in } \Omega, \\ v(0)=1, \quad v=0 & \text { on } \partial \Omega .\end{cases}
$$

But if $\Omega$ is a half-space, then by [12], $v$ must vanish identically and thus we derive a contradiction. If $\Omega$ is a strip in $\mathbb{R}^{n}$ and condition $\left(H_{1}\right)$ is satisfied, then by the Pohozaev identity (see e.g. [24, Theorem III.1.3]), $v \equiv 0$ and thus we also obtain a contradiction in this case. Lastly, if $\Omega$ is a strip in $\mathbb{R}^{n}$ and condition $\left(H_{2}\right)$ is satisfied, then by Theorem 3 . we also find a contradiction. Thus our lemma follows. 
From Lemma 5, we derive that there exists some positive function $v$ such that (after passing to a subsequence) $v_{\varepsilon} \rightarrow v$ in $C_{\mathrm{loc}}^{1}\left(\mathbb{R}^{n}\right)$, and $v$ satisfies

$$
\left\{\begin{array}{l}
-\Delta v=v^{(n+2) /(n-2)}, \quad v>0 \quad \text { in } \mathbb{R}^{n}, \\
v(0)=1, \quad \nabla v(0)=0 .
\end{array}\right.
$$

It follows from [9] that

$$
v(X)=\delta_{\left(0, \alpha_{n}\right)}(X),
$$

where $\alpha_{n}=(n(n-2))^{-1 / 2}$ and, for $a \in \mathbb{R}^{n}$ and $\lambda>0$,

$$
\delta_{(a, \lambda)}(x)=c_{0} \frac{\lambda^{(n-2) / 2}}{\left(1+\lambda^{2}|x-a|^{2}\right)^{(n-2) / 2}} \quad \text { with } \quad c_{0}=(n(n-2))^{(n-2) / 4} .
$$

We recall that $\delta_{(a, \lambda)}$ are the only minimizers for the Sobolev constant

$$
S=\inf \left\{\|\nabla u\|_{L^{2}\left(\mathbb{R}^{n}\right)}^{2}\|u\|_{L^{2 n /(n-2)}\left(\mathbb{R}^{n}\right)}^{-2}:|\nabla u| \in L^{2}, u \in L^{2 n /(n-2)}, u \neq 0\right\} .
$$

We note that, by the above arguments, for any $R>0$ we have

$$
\int_{B\left(a_{1, \varepsilon}, R / \lambda_{1, \varepsilon}\right)} u_{\varepsilon}^{2 n /(n-2)}(x) d x \rightarrow \int_{B(0, R)} \delta_{\left(0, \alpha_{n}\right)}^{2 n /(n-2)}(x) d x \quad \text { as } \varepsilon \rightarrow 0,
$$

where $\lambda_{1, \varepsilon}=M_{1, \varepsilon}^{2 /(n-2)}$.

To proceed further, we introduce the function

$$
\tilde{u}_{\varepsilon}(X)=d_{1, \varepsilon}^{(n-2) / 2} u_{\varepsilon}\left(a_{1, \varepsilon}+d_{1, \varepsilon} X\right), \quad X \in \tilde{A}_{\varepsilon}:=d_{1, \varepsilon}^{-1}\left(A_{\varepsilon}-a_{1, \varepsilon}\right),
$$

where $d_{1, \varepsilon}=d\left(a_{1, \varepsilon}, \partial A_{\varepsilon}\right)$. Notice that from Lemma 5, we know that

$$
\tilde{u}_{\varepsilon}(0)=d_{1, \varepsilon}^{(n-2) / 2} u_{\varepsilon}\left(a_{1, \varepsilon}\right) \rightarrow+\infty \quad \text { as } \varepsilon \rightarrow 0 .
$$

We observe that the limit domain of $\tilde{A}_{\varepsilon}$ is a strip or a half-space in $\mathbb{R}^{n}$; we denote it by $\Pi$ in both cases.

As a first step of our blow up process, we prove the following proposition.

Proposition 6. We have

$$
h_{\varepsilon}:=\max _{x \in A_{\varepsilon}}\left(\left|x-a_{1, \varepsilon}\right|^{(n-2) / 2} u_{\varepsilon}(x)\right) \rightarrow+\infty \quad \text { as } \varepsilon \rightarrow 0 .
$$

Proof. Arguing by contradiction, we suppose that $h_{\varepsilon} \leq C$, with $C$ is a positive constant independent of $\varepsilon$. Thus, we have

$$
|X|^{(n-2) / 2} \tilde{u}_{\varepsilon}(X) \leq C, \quad \forall X \in \tilde{A}_{\varepsilon} .
$$

In particular,

$$
\left\{\begin{array}{l}
\tilde{u}_{\varepsilon}(X) \leq C|X|^{(2-n) / 2}, \quad \forall X \in B(0,1 / 2) \backslash\{0\} \\
\tilde{u}_{\varepsilon}(0) \rightarrow+\infty
\end{array}\right.
$$


Therefore 0 is an isolated blow up point of $\tilde{u}_{\varepsilon}$ (see Appendix for definition). Then it follows from Proposition 19 that 0 is an isolated simple blow up point (see Appendix) in $B(0,1 / 2)$. Applying Proposition 18 of the Appendix we derive that there exist positive constants $c_{1}$ and $c_{2}$ such that

$$
\frac{c_{1} \tilde{u}_{\varepsilon}(0)}{\left(1+\tilde{u}_{\varepsilon}^{4 /(n-2)}(0)|y|^{2}\right)^{(n-2) / 2}} \leq \tilde{u}_{\varepsilon}(y) \leq \frac{c_{2}}{\tilde{u}_{\varepsilon}(0)|y|^{n-2}}, \quad \text { for } y \in B(0,1 / 4) \backslash\{0\} .
$$

Considering now the linear equation

$$
\Delta u+V u=0 \quad \text { with } \quad V=\tilde{u}_{\varepsilon}^{4 /(n-2)},
$$

we deduce from Lemma 16 and the Harnack inequality (see [13]) that

$$
\tilde{u}_{\varepsilon}(y) \leq c_{K} \tilde{u}_{\varepsilon}(0)^{-1}, \quad \forall y \in K,
$$

where $K$ is any compact subset of $\tilde{A}_{\varepsilon}$ which does not contain 0 , and $c_{K}$ is a constant depending on $K$.

Now we set

$$
\tilde{v}_{\varepsilon}(X)=\tilde{u}_{\varepsilon}(0) \tilde{u}_{\varepsilon}(X) .
$$

It is easy to check that $\tilde{v}_{\varepsilon}$ satisfies

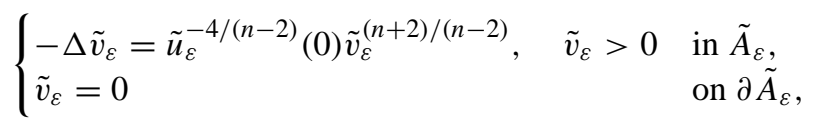

and

$$
\begin{gathered}
\tilde{v}_{\varepsilon}(0) \rightarrow+\infty \quad \text { as } \varepsilon \rightarrow 0, \\
c_{1}|y|^{2-n} \leq \tilde{v}_{\varepsilon}(y) \leq c_{2}|y|^{2-n}, \quad \forall y \in K,
\end{gathered}
$$

where $K$ is any compact subset of $\tilde{A}_{\varepsilon} \backslash\{0\}$. It follows from standard elliptic theory that

$$
\tilde{v}_{\varepsilon} \rightarrow \alpha G_{\Pi}(0, \cdot) \quad \text { in } C_{\mathrm{loc}}^{2}(\Pi),
$$

where $G_{\Pi}(0, \cdot)$ is the Green function of the Laplace operator with Dirichlet boundary condition defined on the limit domain $\Pi$ (half-space or strip) and where $\alpha$ is a positive constant. Such a Green function can be written as

$$
G_{\Pi}(0, x)=|x|^{2-n}-H(0, x),
$$

where $H(0, x)>0$ by the maximum principle. We now observe that $\tilde{u}_{\varepsilon}$ satisfies

$$
-\Delta \tilde{u}_{\varepsilon}=\tilde{u}_{\varepsilon}^{(n+2) /(n-2)} \quad \text { in } B_{r}:=B(0, r) \text { for any } r<1 / 2 \text {. }
$$

Applying the Pohozaev identity (see for example Corollary 1.1 of [17]), we derive that

$$
-r \frac{n-2}{2 n} \int_{\partial B_{r}} \tilde{u}_{\varepsilon}^{2 n /(n-2)}=\int_{\partial B_{r}} B\left(r, x, \tilde{u}_{\varepsilon}, \nabla \tilde{u}_{\varepsilon}\right),
$$


where

$$
B\left(r, x, \tilde{u}_{\varepsilon}, \nabla \tilde{u}_{\varepsilon}\right)=\frac{n-2}{2} \tilde{u}_{\varepsilon} \frac{\partial \tilde{u}_{\varepsilon}}{\partial v}-\frac{r}{2}\left|\nabla \tilde{u}_{\varepsilon}\right|^{2}+r\left(\frac{\partial \tilde{u}_{\varepsilon}}{\partial v}\right)^{2} .
$$

On the one hand, using 2.8, we obtain

$$
r \frac{n-2}{2 n} \int_{\partial B_{r}} \tilde{u}_{\varepsilon}^{2 n /(n-2)} \leq c \frac{\tilde{u}_{\varepsilon}^{-2 n /(n-2)}(0)}{r^{n}} .
$$

Multiplying 2.9] by $\tilde{u}_{\varepsilon}^{2}(0)$, we derive that

$$
\tilde{u}_{\varepsilon}(0)^{2} \int_{\partial B_{r}} B\left(r, x, \tilde{u}_{\varepsilon}, \nabla \tilde{u}_{\varepsilon}\right)=O\left(\frac{\tilde{u}_{\varepsilon}^{-4 /(n-2)}(0)}{r^{n}}\right) .
$$

Using the homogeneity of the operator $B$, we obtain

$$
\int_{\partial B_{r}} B\left(r, x, \tilde{v}_{\varepsilon}, \nabla \tilde{v}_{\varepsilon}\right)=O\left(\frac{\tilde{u}_{\varepsilon}^{-4 /(n-2)}(0)}{r^{n}}\right) .
$$

In particular, we conclude that

$$
\lim _{\varepsilon \rightarrow 0} \int_{\partial B_{r}} B\left(r, x, \tilde{v}_{\varepsilon}, \nabla \tilde{v}_{\varepsilon}\right)=0 \quad \text { for } 0<r<1 / 2 .
$$

On the other hand,

$$
\tilde{v}_{\varepsilon} \rightarrow \alpha G_{\Pi}(0, \cdot) \quad \text { in } C^{2}\left(\partial B_{r}\right), \text { for } 0<r<1 / 2,
$$

and for $r$ small enough,

$$
G_{\Pi}(0, x)=|x|^{2-n}-H(0,0)+o(|x|), \quad \text { with }|x|=r .
$$

Thus we have

$$
\lim _{\varepsilon \rightarrow 0, r \rightarrow 0} \int_{\partial B_{r}} B\left(r, x, \tilde{v}_{\varepsilon}, \nabla \tilde{v}_{\varepsilon}\right)=\frac{(n-2)^{2}}{2} H(0,0) \alpha^{2}\left|S^{n-1}\right|>0,
$$

which contradicts 2.10 , and thus our proposition follows.

Let $a_{2, \varepsilon} \in A_{\varepsilon}$ be such that

$$
h_{\varepsilon}=\left|a_{2, \varepsilon}-a_{1, \varepsilon}\right|^{(n-2) / 2} u_{\varepsilon}\left(a_{2, \varepsilon}\right),
$$

where $h_{\varepsilon}$ is defined in Proposition 6. Now if we blow up at the point $a_{2, \varepsilon}$, Proposition 6 implies that the image under the new scaling of the first point $a_{1, \varepsilon}$ will escape to infinity, a fact that we express loosely by saying that these points ignore themselves. However the domain may become degenerate, that is, its width becomes thinner and thinner along the blow up process. The following lemma rules out such a situation. 
Lemma 7. There exists $\delta>0$ such that for every $\varepsilon$,

$$
\lambda_{2, \varepsilon} \varepsilon \geq \delta
$$

where $\lambda_{2, \varepsilon}=u_{\varepsilon}^{2 /(n-2)}\left(a_{2, \varepsilon}\right)$.

Proof. For $X \in B\left(0,\left(\lambda_{2, \varepsilon} / 2\right)\left|a_{1, \varepsilon}-a_{2, \varepsilon}\right|\right) \cap D_{\varepsilon}$, we set

$$
w_{\varepsilon}(X)=\lambda_{2, \varepsilon}^{(2-n) / 2} u_{\varepsilon}\left(a_{2, \varepsilon}+\lambda_{2, \varepsilon}^{-1} X\right), \quad \text { with } \quad D_{\varepsilon}=\lambda_{2, \varepsilon}\left(A_{\varepsilon}-a_{2, \varepsilon}\right) .
$$

Recall that, for any $x \in A_{\varepsilon}$, we have

$$
\left|x-a_{1, \varepsilon}\right|^{(n-2) / 2} u_{\varepsilon}(x) \leq\left|a_{2, \varepsilon}-a_{1, \varepsilon}\right|^{(n-2) / 2} u_{\varepsilon}\left(a_{2, \varepsilon}\right)=\left|a_{2, \varepsilon}-a_{1, \varepsilon}\right|^{(n-2) / 2} \lambda_{2, \varepsilon}^{(n-2) / 2} .
$$

Thus, for any $x \in A_{\varepsilon}$, we obtain

$$
\frac{u_{\varepsilon}(x)}{\lambda_{2, \varepsilon}^{(n-2) / 2}} \leq \frac{\left|a_{2, \varepsilon}-a_{1, \varepsilon}\right|^{(n-2) / 2}}{\left|x-a_{1, \varepsilon}\right|^{(n-2) / 2}} .
$$

But, for $x \in B\left(a_{2, \varepsilon},\left|a_{2, \varepsilon}-a_{1, \varepsilon}\right| / 2\right)$, we have $\left|x-a_{1, \varepsilon}\right| \geq\left|a_{2, \varepsilon}-a_{1, \varepsilon}\right| / 2$. Hence

$$
\frac{u_{\varepsilon}(x)}{\lambda_{2, \varepsilon}^{(n-2) / 2}} \leq 2^{(n-2) / 2} \quad \text { for any } x \in B\left(a_{2, \varepsilon},\left|a_{2, \varepsilon}-a_{1, \varepsilon}\right| / 2\right) \text {. }
$$

Thus we obtain

$$
w_{\varepsilon}(X) \leq 2^{n-2 / 2}, \quad \forall X \in B\left(0,\left(\lambda_{2, \varepsilon} / 2\right)\left|a_{1, \varepsilon}-a_{2, \varepsilon}\right|\right) \cap D_{\varepsilon} .
$$

Arguing by contradiction, we suppose that

$$
\lambda_{2, \varepsilon} \varepsilon \rightarrow 0 \quad \text { as } \varepsilon \rightarrow 0
$$

Let $\bar{a}_{2, \varepsilon} \in \partial D_{\varepsilon}$ be such that $\left|\bar{a}_{2, \varepsilon}\right|=d\left(0, \partial D_{\varepsilon}\right)$. We may assume without loss of generality that the unit outward normal to $\partial D_{\varepsilon}$ at $\bar{a}_{2, \varepsilon}$ is $e_{n}$, where $e_{n}$ is the $n$th element of the canonical basis of $\mathbb{R}^{n}$. Let

$$
B\left(\bar{a}_{2, \varepsilon}^{\prime}, 1\right)=\left\{x^{\prime} \in \mathbb{R}^{n-1}:\left|x^{\prime}-\bar{a}_{2, \varepsilon}^{\prime}\right|<1\right\},
$$

where

$$
\bar{a}_{2, \varepsilon}=\left(\bar{a}_{2, \varepsilon}^{\prime}, \bar{a}_{2, \varepsilon}^{n}\right) \in \mathbb{R}^{n-1} \times \mathbb{R}, \quad x=\left(x^{\prime}, x^{n}\right) \in \mathbb{R}^{n-1} \times \mathbb{R}
$$

Let

$$
\begin{gathered}
T_{\varepsilon}=\left(B\left(\bar{a}_{2, \varepsilon}^{\prime}, 1\right) \times[-1,1]\right) \cap D_{\varepsilon}, \\
\partial T_{\varepsilon}^{1}=\partial\left(T_{\varepsilon}\right) \cap \partial D_{\varepsilon}, \quad \partial T_{\varepsilon}^{2}=\partial\left(T_{\varepsilon}\right) \cap D_{\varepsilon} .
\end{gathered}
$$


We denote by $G_{T_{\varepsilon}}$ the Green function of the Laplace operator with Dirichlet boundary condition defined on $T_{\varepsilon}$. Let $X \in T_{\varepsilon}$ such that $X=\beta_{\varepsilon} e_{n}$, with $-1 \leq \beta_{\varepsilon} \leq 1$. By easy computations, one can check that

$$
\int_{T_{\varepsilon}} G_{T_{\varepsilon}}(X, y) d y \leq \int_{T_{\varepsilon}} \frac{d y}{|X-y|^{n-2}}=O\left(\lambda_{2, \varepsilon} \varepsilon\right) .
$$

Now we observe that

$$
\begin{aligned}
w_{\varepsilon}(X) & =c_{n}\left(\int_{T_{\varepsilon}} G_{T_{\varepsilon}}(X, y) w_{\varepsilon}^{(n+2) /(n-2)}(y) d y-\int_{\partial T_{\varepsilon}} \frac{\partial G_{T_{\varepsilon}}}{\partial v}(X, y) w_{\varepsilon}(y) d y\right) \\
& =c_{n}\left(\int_{T_{\varepsilon}} G_{T_{\varepsilon}}(X, y) w_{\varepsilon}^{(n+2) /(n-2)}(y) d y-\int_{\partial T_{\varepsilon}^{2}} \frac{\partial G_{T_{\varepsilon}}}{\partial v}(X, y) w_{\varepsilon}(y) d y\right),
\end{aligned}
$$

where $c_{n}$ is a positive constant. But, since $X=\beta_{\varepsilon} e_{n}$, we have

$$
\frac{\partial G_{T_{\varepsilon}}}{\partial v}(X, y) \leq c, \quad \forall y \in \partial T_{\varepsilon}^{2}
$$

Since $w_{\varepsilon} \leq 2^{(n-2) / 2}$, we derive that

$\int_{T_{\varepsilon}} G_{T_{\varepsilon}}(X, y) w_{\varepsilon}^{(n+2) /(n-2)}(y) d y=O\left(\lambda_{2, \varepsilon} \varepsilon\right), \quad \int_{\partial T_{\varepsilon}^{2}} \frac{\partial G_{T_{\varepsilon}}}{\partial v}(X, y) w_{\varepsilon}(y) d y=O\left(\lambda_{2, \varepsilon} \varepsilon\right)$.

Thus we obtain $w_{\varepsilon}(X)=O\left(\lambda_{2, \varepsilon} \varepsilon\right)$, and in particular $w_{\varepsilon}(0)=1 \leq c \lambda_{2, \varepsilon} \varepsilon$. Thus we derive a contradiction and therefore our lemma follows.

Now, since $\lambda_{2, \varepsilon} \varepsilon \not \supset 0$ as $\varepsilon \rightarrow 0$, we can prove, as in Lemma 5 , that

$$
\lambda_{2, \varepsilon} d\left(a_{2, \varepsilon}, \partial A_{\varepsilon}\right) \rightarrow+\infty \quad \text { as } \varepsilon \rightarrow 0
$$

and therefore there exist $b \in \mathbb{R}^{n}$ and $\mu>0$ such that the function $w_{\varepsilon}$ defined by 2.11] converges in $C_{\text {loc }}^{1}\left(\mathbb{R}^{n}\right)$ to $\delta_{(b, \mu)}$. Thus we have found a second blow up point $\bar{a}_{2, \varepsilon}$ of $u_{\varepsilon}$ with concentration $\bar{\lambda}_{2, \varepsilon}$ defined by

$$
\bar{a}_{2, \varepsilon}=a_{2, \varepsilon}+\frac{b}{\lambda_{2, \varepsilon}}, \quad \bar{\lambda}_{2, \varepsilon}=\mu \lambda_{2, \varepsilon} .
$$

Observe that $\bar{\lambda}_{2, \varepsilon} \varepsilon=\mu \lambda_{2, \varepsilon} \varepsilon \nrightarrow 0$ as $\varepsilon \rightarrow 0$, and therefore as above we have

$$
\bar{\lambda}_{2, \varepsilon} d\left(\bar{a}_{2, \varepsilon}, \partial A_{\varepsilon}\right) \rightarrow+\infty \quad \text { as } \varepsilon \rightarrow 0 \text {. }
$$

Summarizing, we have built two points $a_{1, \varepsilon}, \bar{a}_{2, \varepsilon}$ with concentrations $\lambda_{1, \varepsilon}$ and $\bar{\lambda}_{2, \varepsilon}$ such that, as $\varepsilon \rightarrow 0$,

$$
\begin{aligned}
& \lambda_{1, \varepsilon} d\left(a_{1, \varepsilon}, \partial A_{\varepsilon}\right) \rightarrow+\infty, \quad \bar{\lambda}_{2, \varepsilon} d\left(\bar{a}_{2, \varepsilon}, \partial A_{\varepsilon}\right) \rightarrow+\infty, \\
& \forall R>0 \quad \int_{B\left(a_{1, \varepsilon}, R / \lambda_{1, \varepsilon}\right)} u_{\varepsilon}^{2 n /(n-2)}(x) d x \rightarrow \int_{B(0, R)} \delta_{\left(0, \alpha_{n}\right)}^{2 n /(n-2)}(x) d x, \\
& \forall R>0 \quad \int_{B\left(\bar{a}_{2, \varepsilon}, R / \bar{\lambda}_{2, \varepsilon}\right)} u_{\varepsilon}^{2 n /(n-2)}(x) d x \rightarrow \int_{B(b, R / \mu)} \delta_{(b, \mu)}^{2 n /(n-2)}(x) d x, \\
& \left|a_{1, \varepsilon}-\bar{a}_{2, \varepsilon}\right| \lambda_{1, \varepsilon} \rightarrow+\infty, \quad\left|a_{1, \varepsilon}-\bar{a}_{2, \varepsilon}\right| \bar{\lambda}_{2, \varepsilon} \rightarrow+\infty .
\end{aligned}
$$


In this section, we have started a blow up process, producing blow up points which ignore each other, and therefore contribute to the total energy at least a fixed amount. Our goal now is to prove that the process does not stop after finitely many steps. This is a key argument in the proof of Theorem 1 . See Proposition 8 in the next section for a quantitative statement of this fact.

\section{Proof of Theorem 1}

This section is devoted to the proof of Theorem 1 . We first prove that the process started in Section 2 does not stop after finitely many steps Actually we have:

Proposition 8. Let $\mathcal{S}=\left\{x_{1}^{\varepsilon}, \ldots, x_{p}^{\varepsilon}\right\}, p \geq 2$, be such that, as $\varepsilon \rightarrow 0$,

$$
\begin{aligned}
d\left(x_{i}^{\varepsilon}, \partial A_{\varepsilon}\right)^{n-2 /(2)} u_{\varepsilon}\left(x_{i}^{\varepsilon}\right) \rightarrow \infty & \text { for } 1 \leq i \leq p, \\
\left|x_{i}^{\varepsilon}-x_{j}^{\varepsilon}\right|^{(n-2) / 2} u_{\varepsilon}\left(x_{j}^{\varepsilon}\right) \rightarrow \infty & \text { for } i \neq j, 1 \leq i, j \leq p .
\end{aligned}
$$

Then

$$
\max _{x \in A_{\varepsilon}} d(x, \mathcal{S})^{(n-2) / 2} u_{\varepsilon}(x) \rightarrow \infty \quad \text { as } \varepsilon \rightarrow 0 .
$$

Proof. Arguing by contradiction, we assume that there exists $C>0$ such that

$$
d(x, \mathcal{S})^{(n-2) / 2} u_{\varepsilon}(x)<C, \quad \forall x \in A_{\varepsilon} .
$$

Without loss of generality, we may assume that

$$
d\left(x_{p}^{\varepsilon}, \partial A_{\varepsilon}\right)=\min _{1 \leq i \leq p} d\left(x_{i}^{\varepsilon}, \partial A_{\varepsilon}\right) .
$$

We set

$$
d_{p}^{\varepsilon}=d\left(x_{p}^{\varepsilon}, \partial A_{\varepsilon}\right) ; \quad w_{\varepsilon}(X)=\left(d_{p}^{\varepsilon}\right)^{(n-2) / 2} u_{\varepsilon}\left(d_{p}^{\varepsilon} X+x_{p}^{\varepsilon}\right) ; \quad X_{j}^{\varepsilon}=\frac{x_{j}^{\varepsilon}-x_{p}^{\varepsilon}}{d_{p}^{\varepsilon}} .
$$

Observe that $X_{p}^{\varepsilon}=0$. We distinguish two cases:

Case 1: $\min _{1 \leq i \leq p-1}\left|X_{i}^{\varepsilon}\right| \leq \min _{i \neq j}\left|X_{i}^{\varepsilon}-X_{j}^{\varepsilon}\right|$. In this case we prove the following lemma:

Lemma 9. There exists $\delta>0$ such that $\left|X_{i}^{\varepsilon}\right| \geq \delta$ for $i \in\{1, \ldots, p-1\}$.

Proof. Without loss of generality we may assume that $\left|X_{1}^{\varepsilon}\right|=\min _{1 \leq i \leq p-1}\left|X_{i}^{\varepsilon}\right|$. Arguing by contradiction, we suppose that $\tau:=\left|X_{1}^{\varepsilon}\right| \rightarrow 0$. Consider

$$
\tilde{w}_{\varepsilon}(\tilde{X}):=\tau^{(n-2) / 2} w_{\varepsilon}(\tau \tilde{X}), \quad \text { where } \quad \tilde{X}=X / \tau \text {, so that }\left|\tilde{X}_{1}^{\varepsilon}\right|=1 .
$$

Observe that

$$
d\left(X, \mathcal{S}_{1}\right)^{(n-2) / 2} w_{\varepsilon}(X) \leq C, \quad \text { where } \quad \mathcal{S}_{1}=\left\{0, X_{1}^{\varepsilon}, \ldots, X_{p-1}^{\varepsilon}\right\},
$$


implies that

$$
\begin{cases}|y|^{(n-2) / 2} w_{\varepsilon}(y) \leq C & \text { for all }|y| \leq \frac{1}{2} \tau \\ \left|y-X_{1}^{\varepsilon}\right|^{(n-2) / 2} w_{\varepsilon}(y) \leq C & \text { for all }\left|y-X_{1}^{\varepsilon}\right| \leq \frac{1}{2} \tau .\end{cases}
$$

It follows that

$$
\begin{cases}|y|^{(n-2) / 2} \tilde{w}_{\varepsilon}(y) \leq C & \text { for all }|y| \leq \frac{1}{2} \\ \left|y-\tilde{X}_{1}^{\varepsilon}\right|^{(n-2) / 2} \tilde{w}_{\varepsilon}(y) \leq C & \text { for all }\left|y-\tilde{X}_{1}^{\varepsilon}\right| \leq \frac{1}{2}\end{cases}
$$

Notice that

$$
\begin{gathered}
\tilde{w}_{\varepsilon}(0)=\tau^{(n-2) / 2} w_{\varepsilon}(0)=\left|x_{1}^{\varepsilon}-x_{p}^{\varepsilon}\right|^{(n-2) / 2} u_{\varepsilon}\left(x_{p}^{\varepsilon}\right) \rightarrow \infty, \\
\tilde{w}_{\varepsilon}\left(\tilde{X}_{1}^{\varepsilon}\right)=\left|x_{1}^{\varepsilon}-x_{p}^{\varepsilon}\right|^{(n-2) / 2} u_{\varepsilon}\left(x_{1}^{\varepsilon}\right) \rightarrow \infty .
\end{gathered}
$$

It follows that 0 and $\tilde{X}_{1}:=\lim _{\varepsilon \rightarrow 0} \tilde{X}_{1}^{\varepsilon}$ are isolated simple blow up points (see Appendix). Now it follows from standard elliptic theory and properties of isolated simple blow up points that

$$
\left\{\begin{array}{l}
\lim _{\varepsilon \rightarrow 0} \tilde{w}_{\varepsilon}(0) \tilde{w}_{\varepsilon}(y)=h(y) \quad \text { in } C_{\mathrm{loc}}^{0}\left(\mathbb{R}^{n} \backslash \tilde{\mathcal{S}}_{2}\right), \\
h(y)>0, \quad y \in \mathbb{R}^{n} \backslash \tilde{\mathcal{S}}_{2},
\end{array}\right.
$$

where $h$ is harmonic outside its singular set $\tilde{\mathcal{S}}_{2} \subset \mathcal{S}_{2}$, and $\mathcal{S}_{2}=\left\{0, \tilde{X}_{1}, \ldots, \tilde{X}_{p-1}\right\}$, with $\tilde{X}_{i}=\lim _{\varepsilon \rightarrow 0} \tilde{X}_{i}^{\varepsilon}$ for $1 \leq i \leq p-1$. Observing that $\left(0, \tilde{X}_{1}\right) \in \tilde{\mathcal{S}}_{2}$, we then deduce from Böcher's theorem (see e.g. [15]) and the maximum principle that there exist some nonnegative function $b(y)$ and positive constants $M_{1}, M_{2}$ such that for all $y \in \mathbb{R}^{n} \backslash$ $\left\{\tilde{\mathcal{S}}_{2} \backslash\left\{0, \tilde{X}_{1}\right\}\right\}$,

$$
\left\{\begin{array}{l}
b(y) \geq 0, \\
\Delta b(y)=0, \\
h(y)=M_{1}|y|^{2-n}+M_{2}\left|y-\tilde{X}_{1}\right|^{2-n}+b(y) .
\end{array}\right.
$$

Therefore for some constant $A>0$,

$$
h(y)=M_{1}|y|^{2-n}+A+O(|y|) \quad \text { for } y \text { close to } 0 .
$$

As usual we derive a contradiction like in the proof of Proposition 6 The proof of Lemma 9 is thereby completed.

Case 2: $\min _{1 \leq i \leq p-1}\left|X_{i}^{\varepsilon}\right|>\min _{i \neq j}\left|X_{i}^{\varepsilon}-X_{j}^{\varepsilon}\right|$.

Lemma 10. There exists $\delta>0$ such that

$$
\min _{i \neq j}\left|X_{i}^{\varepsilon}-X_{j}^{\varepsilon}\right| \geq \delta \quad \text { for } i, j \in\{1, \ldots, p-1\} .
$$


Proof. Without loss of generality, we may assume that

$$
\left|X_{1}^{\varepsilon}-X_{2}^{\varepsilon}\right|=\min _{i \neq j}\left|X_{i}^{\varepsilon}-X_{j}^{\varepsilon}\right| .
$$

Suppose by contradiction that

$$
\sigma_{\varepsilon}:=\left|X_{1}^{\varepsilon}-X_{2}^{\varepsilon}\right| \rightarrow 0
$$

and set

$$
v_{\varepsilon}(y)=\sigma^{n-2 / 2} w_{\varepsilon}\left(\sigma_{\varepsilon} y+X_{1}^{\varepsilon}\right)
$$

It follows that $v_{\varepsilon}$ satisfies

$$
-\Delta v_{\varepsilon}=v_{\varepsilon}^{(n+2) /(n-2)}, \quad v_{\varepsilon}>0 \quad \text { in }|y| \leq 1 / \sigma_{\varepsilon} .
$$

Taking $Y_{2}^{\varepsilon}$ such that $X_{2}^{\varepsilon}=\sigma_{\varepsilon} Y_{2}^{\varepsilon}+X_{1}^{\varepsilon}$, it is easy to see that

$$
\begin{array}{lll}
v_{\varepsilon}(y) \leq C|y|^{-(n-2) / 2} & \text { for all }|y| \leq \frac{1}{2}, & v_{\varepsilon}(0) \rightarrow \infty \\
v_{\varepsilon}(y) \leq C\left|y-Y_{2}^{\varepsilon}\right|^{-(n-2) / 2} & \text { for all }\left|y-Y_{2}^{\varepsilon}\right| \leq \frac{1}{2}, & v_{\varepsilon}\left(Y_{2}^{\varepsilon}\right) \rightarrow \infty
\end{array}
$$

It follows that 0 and $Y_{2}:=\lim _{\varepsilon \rightarrow 0} Y_{2}^{\varepsilon}$ are isolated simple blow up points, therefore arguing as in the first case, we derive a contradiction.

Coming back to the proof of Proposition 8, we see that, from Lemmas 9 and 10 , there exists $\delta>0$, which does not depend on $\varepsilon$, such that:

$$
\begin{cases}|X|^{(n-2) / 2} w_{\varepsilon}(X) \leq C & \text { for every } X \in B(0, \delta / 2) \\ \left|X-X_{1}^{\varepsilon}\right|^{(n-2) / 2} w_{\varepsilon}(X) \leq C & \text { for every } X \in B\left(X_{1}^{\varepsilon}, \delta / 2\right), \\ w_{\varepsilon}(0)=\left(d_{p}^{\varepsilon}\right)^{(n-2) / 2} u_{\varepsilon}\left(x_{p}^{\varepsilon}\right) \rightarrow \infty & \end{cases}
$$

We distinguish two cases:

- $\left|X_{1}^{\varepsilon}\right| \rightarrow \infty$ as $\varepsilon \rightarrow 0$. In this case 0 is the only isolated blow up point of $w_{\varepsilon}$ and thus, arguing as in the proof of Proposition 6, we derive a contradiction.

- There exists a constant $C>0$ such that $\left|X_{1}^{\varepsilon}\right| \leq C$. In this case we argue as in the proof of Lemma 9 or Lemma 10 to derive a contradiction.

The proof of Proposition 8 is thereby completed.

In the next proposition, we prove that at each blow up point constructed by our blow up scheme, the projection on $H_{0}^{1}\left(A_{\varepsilon}\right)$ of the bubble concentrating there contributes at least 1 to the total Morse index of $u_{\varepsilon}$.

Proposition 11. Let $\left\{x_{1}^{\varepsilon}, \ldots, x_{p}^{\varepsilon}\right\}, p \geq 2$, be such that, as $\varepsilon \rightarrow 0$,

$$
\begin{aligned}
d\left(x_{i}^{\varepsilon}, \partial A_{\varepsilon}\right)^{(n-2) / 2} u_{\varepsilon}\left(x_{i}^{\varepsilon}\right) \rightarrow \infty & \text { for } 1 \leq i \leq p, \\
\left|x_{i}^{\varepsilon}-x_{j}^{\varepsilon}\right|^{(n-2) / 2} u_{\varepsilon}\left(x_{j}^{\varepsilon}\right) \rightarrow \infty & \text { for } i \neq j, 1 \leq i, j \leq p .
\end{aligned}
$$

Then $m\left(u_{\varepsilon}\right) \geq p$. 
Proof. We begin by introducing some notation. We denote by $q$ the quadratic form associated to the linearized operator $-\Delta-\frac{n+2}{n-2} u_{\varepsilon}^{4 /(n-2)}$ defined on $H_{0}^{1}\left(A_{\varepsilon}\right) \cap H^{2}\left(A_{\varepsilon}\right)$. Thus, for $v \in H_{0}^{1}\left(A_{\varepsilon}\right) \cap H^{2}\left(A_{\varepsilon}\right)$, we have

$$
q(v)=\int_{A_{\varepsilon}}|\nabla v|^{2}-\frac{n+2}{n-2} \int_{A_{\varepsilon}} u_{\varepsilon}^{4 /(n-2)} v^{2} .
$$

Let

$$
d_{i}^{\prime}=\min \left\{d\left(x_{i}^{\varepsilon}, \partial A_{\varepsilon}\right), \frac{1}{2}\left|x_{i}^{\varepsilon}-x_{j}^{\varepsilon}\right| \text { for } j \neq i\right\},
$$

and denote by $P_{\varepsilon} \delta_{\left(x_{i}^{\varepsilon}, \lambda_{i}^{\varepsilon}\right)}$ the projection of $\delta_{\left(x_{i}^{\varepsilon}, \lambda_{i}^{\varepsilon}\right)}$ onto $H_{0}^{1}\left(B\left(x_{i}^{\varepsilon}, d_{i}^{\prime}\right)\right)$, that is,

$$
\Delta P_{\varepsilon} \delta_{\left(x_{i}^{\varepsilon}, \lambda_{i}^{\varepsilon}\right)}=\Delta \delta_{\left(x_{i}^{\varepsilon}, \lambda_{i}^{\varepsilon}\right)} \quad \text { in } B\left(x_{i}^{\varepsilon}, d_{i}^{\prime}\right), \quad P_{\varepsilon} \delta_{\left(x_{i}^{\varepsilon}, \lambda_{i}^{\varepsilon}\right)}=0 \quad \text { on } \partial B\left(x_{i}^{\varepsilon}, d_{i}^{\prime}\right),
$$

$P_{\varepsilon} \delta_{\left(x_{i}^{\varepsilon}, \lambda_{i}^{\varepsilon}\right)}$ being continued by 0 in $A_{\varepsilon} \backslash B\left(x_{i}^{\varepsilon}, d_{i}^{\prime}\right)$ (here and below, $\left.\lambda_{i}^{\varepsilon}=\left(u_{\varepsilon}\left(x_{i}^{\varepsilon}\right)\right)^{2 /(n-2)}\right)$. Clearly the supports of the functions $P_{\varepsilon} \delta_{\left(x_{i}^{\varepsilon}, \lambda_{i}^{\varepsilon}\right)}$ are disjoint and $\lambda_{i}^{\varepsilon} d_{i}^{\prime} \rightarrow+\infty$ for each $i$.

In order to prove our proposition, it is sufficient to prove that, for $\varepsilon$ small,

$$
q\left(\sum_{i=1}^{p} \alpha_{i} P_{\varepsilon} \delta_{\left(x_{i}^{\varepsilon}, \lambda_{i}^{\varepsilon}\right)}\right)<0 \quad \forall \alpha_{i} \in \mathbb{R} .
$$

To simplify our notation we will write $P_{\varepsilon} \delta_{i}$ and $\delta_{i}$ instead of $P_{\varepsilon} \delta_{\left(x_{i}^{\varepsilon}, \lambda_{i}^{\varepsilon}\right)}$ and $\delta_{\left(x_{i}^{\varepsilon}, \lambda_{i}^{\varepsilon}\right)}$ respectively. Now, we observe that

$$
\begin{aligned}
q\left(\sum_{i=1}^{p} \alpha_{i} P_{\varepsilon} \delta_{i}\right)= & \sum_{i=1}^{p} \alpha_{i}^{2} \int_{A_{\varepsilon}}\left|\nabla P_{\varepsilon} \delta_{i}\right|^{2}+\sum_{i \neq j} \alpha_{i} \alpha_{j} \int_{A_{\varepsilon}} \nabla P_{\varepsilon} \delta_{i} \nabla P_{\varepsilon} \delta_{j} \\
& -\frac{n+2}{n-2} \int_{A_{\varepsilon}} u_{\varepsilon}^{4 /(n-2)}\left(\sum_{i=1}^{p} \alpha_{i}^{2} P_{\varepsilon} \delta_{i}^{2}+\sum_{i \neq j} \alpha_{i} \alpha_{j} P_{\varepsilon} \delta_{i} P_{\varepsilon} \delta_{j}\right) \\
= & \sum_{i=1}^{p} \alpha_{i}^{2} q\left(P_{\varepsilon} \delta_{i}\right),
\end{aligned}
$$

where we have used the fact that the supports of the functions $P_{\varepsilon} \delta_{i}$ are disjoint.

We now notice that

$$
\begin{aligned}
q\left(P_{\varepsilon} \delta_{i}\right)= & \int_{A_{\varepsilon}}\left|\nabla P_{\varepsilon} \delta_{i}\right|^{2}-\frac{n+2}{n-2} \int_{B\left(x_{i}^{\varepsilon}, R / \lambda_{i}^{\varepsilon}\right)} u_{\varepsilon}^{4 /(n-2)} P_{\varepsilon} \delta_{i}^{2} \\
& -\frac{n+2}{n-2} \int_{A_{\varepsilon} \backslash B\left(x_{i}^{\varepsilon}, R / \lambda_{i}^{\varepsilon}\right)} u_{\varepsilon}^{4 /(n-2)} P_{\varepsilon} \delta_{i}^{2} \\
\leq & \int_{B\left(x_{i}^{\varepsilon}, d_{i}^{\prime}\right)}\left|\nabla P_{\varepsilon} \delta_{i}\right|^{2}-\frac{n+2}{n-2} \int_{B\left(x_{i}^{\varepsilon}, R / \lambda_{i}^{\varepsilon}\right)} u_{\varepsilon}^{4 /(n-2)} P_{\varepsilon} \delta_{i}^{2},
\end{aligned}
$$

where $R$ is a large positive constant such that $\int_{\mathbb{R}^{n} \backslash B(0, R)} \delta_{\left(0, \alpha_{n}\right)}^{2 n /(n-2)}=o(1)$ with $\alpha_{n}=$ $(n(n-2))^{-1 / 2}$. 
We now write

$$
\begin{aligned}
\int_{B\left(x_{i}^{\varepsilon}, d_{i}^{\prime}\right)}\left|\nabla P_{\varepsilon} \delta_{i}\right|^{2} & =\int_{B\left(x_{i}^{\varepsilon}, d_{i}^{\prime}\right)} \delta_{i}^{(n+2) /(n-2)} P_{\varepsilon} \delta_{i} \\
& =\int_{B\left(x_{i}^{\varepsilon}, d_{i}^{\prime}\right)} \delta_{i}^{2 n /(n-2)}-\int_{B\left(x_{i}^{\varepsilon}, d_{i}^{\prime}\right)} \delta_{i}^{(n+2) /(n-2)}\left(\delta_{i}-P_{\varepsilon} \delta_{i}\right) .
\end{aligned}
$$

For the second integral, we have

$$
\int_{B\left(x_{i}^{\varepsilon}, d_{i}^{\prime}\right)} \delta_{i}^{(n+2) /(n-2)}\left(\delta_{i}-P_{\varepsilon} \delta_{i}\right) \leq c\left|\delta_{i}-P_{\varepsilon} \delta_{i}\right|_{L^{2 n /(n-2)}\left(B\left(x_{i}^{\varepsilon}, d_{i}^{\prime}\right)\right)} \leq c\left(\lambda_{i}^{\varepsilon} d_{i}^{\prime}\right)^{(2-n) / 2} \rightarrow 0
$$

as $\varepsilon \rightarrow 0$; in the last inequality we have used Proposition 1 of [20]. Thus we have

$$
\int_{B\left(x_{i}^{\varepsilon}, d_{i}^{\prime}\right)}\left|\nabla P_{\varepsilon} \delta_{i}\right|^{2}=\int_{B\left(x_{i}^{\varepsilon}, d_{i}^{\prime}\right)} \delta_{i}^{2 n /(n-2)}+o(1) .
$$

We also have

$$
\begin{aligned}
\int_{B\left(x_{i}^{\varepsilon}, R / \lambda_{i}^{\varepsilon}\right)} & u_{\varepsilon}^{4 /(n-2)} P_{\varepsilon} \delta_{i}^{2} \\
& =\int_{B\left(x_{i}^{\varepsilon}, R / \lambda_{i}^{\varepsilon}\right)} \delta_{i}^{4 /(n-2)} P_{\varepsilon} \delta_{i}^{2}+O\left(\int_{B\left(x_{i}^{\varepsilon}, R / \lambda_{i}^{\varepsilon}\right)}\left|u_{\varepsilon}-\delta_{i}\right|^{4 /(n-2)} P_{\varepsilon} \delta_{i}^{2}\right)
\end{aligned}
$$

Thus, since

$$
\begin{gathered}
\int_{B\left(x_{i}^{\varepsilon}, R / \lambda_{i}^{\varepsilon}\right)} \delta_{i}^{4 /(n-2)} P_{\varepsilon} \delta_{i}^{2}=\int_{B\left(x_{i}^{\varepsilon}, R / \lambda_{i}^{\varepsilon}\right)} \delta_{i}^{2 n /(n-2)}+\int_{B\left(x_{i}^{\varepsilon}, R / \lambda_{i}^{\varepsilon}\right)} \delta_{i}^{4 /(n-2)}\left(\delta_{i}-P_{\varepsilon} \delta_{i}\right)^{2} \\
-2 \int_{B\left(x_{i}^{\varepsilon}, R / \lambda_{i}^{\varepsilon}\right)} \delta_{i}^{n+2 / n-2}\left(\delta_{i}-P_{\varepsilon} \delta_{i}\right), \\
\int_{B\left(x_{i}^{\varepsilon}, R / \lambda_{i}^{\varepsilon}\right)} \delta_{i}^{4 /(n-2)}\left(\delta_{i}-P_{\varepsilon} \delta_{i}\right)^{2} \leq c\left|\delta_{i}-P_{\varepsilon} \delta_{i}\right|_{L^{2 n /(n-2)}\left(A_{\varepsilon}\right)}^{2}=o(1),
\end{gathered}
$$

we derive that

$$
\begin{aligned}
\int_{B\left(x_{i}^{\varepsilon}, R / \lambda_{i}^{\varepsilon}\right)} & u_{\varepsilon}^{4 /(n-2)} P_{\varepsilon} \delta_{i}^{2} \\
= & \int_{B\left(x_{i}^{\varepsilon}, R / \lambda_{i}^{\varepsilon}\right)} \delta_{i}^{2 n /(n-2)}+o(1)+O\left(\int_{B\left(x_{i}^{\varepsilon}, R / \lambda_{i}^{\varepsilon}\right)}\left|u_{\varepsilon}-\delta_{i}\right|^{4 /(n-2)} P_{\varepsilon} \delta_{i}^{2}\right) .
\end{aligned}
$$

Therefore we obtain

$$
\begin{aligned}
q\left(P_{\varepsilon} \delta_{i}\right) \leq & \int_{B\left(x_{i}^{\varepsilon}, d_{i}^{\prime}\right)} \delta_{i}^{2 n /(n-2)}-\frac{n+2}{n-2} \int_{B\left(x_{i}^{\varepsilon}, R / \lambda_{i}^{\varepsilon}\right)} \delta_{i}^{2 n /(n-2)} \\
& +O\left(\int_{B\left(x_{i}^{\varepsilon}, R / \lambda_{i}^{\varepsilon}\right)}\left|u_{\varepsilon}-\delta_{i}\right|^{4 /(n-2)} \delta_{i}^{2}\right)+o(1)
\end{aligned}
$$


Now, letting $A_{\varepsilon}^{i}=\lambda_{i}^{\varepsilon}\left(A_{\varepsilon}-x_{i}^{\varepsilon}\right)$ and setting, for $X \in A_{\varepsilon}^{i}$,

$$
v_{i}^{\varepsilon}(X)=\frac{1}{\left(\lambda_{i}^{\varepsilon}\right)^{(n-2) / 2}} u_{\varepsilon}\left(x_{i}^{\varepsilon}+\frac{X}{\lambda_{i}^{\varepsilon}}\right),
$$

we see that $v_{i}^{\varepsilon} \rightarrow \delta_{\left(0, \alpha_{n}\right)}$ in $C_{\text {loc }}^{1}\left(\mathbb{R}^{n}\right)$. Thus 3.7] becomes

$$
\begin{aligned}
q\left(P_{\varepsilon} \delta_{i}\right) \leq & \int_{B\left(0, \lambda_{i}^{\varepsilon} d_{i}^{\prime}\right)} \delta_{\left(0, \alpha_{n}\right)}^{2 n /(n-2)}-\frac{n+2}{n-2} \int_{B(0, R)} \delta_{\left(0, \alpha_{n}\right)}^{2 n /(n-2)} \\
& +O\left(\int_{B(0, R)}\left|v_{i}^{\varepsilon}-\delta_{\left(0, \alpha_{n}\right)}\right|^{4 /(n-2)} \delta_{\left(0, \alpha_{n}\right)}^{2}\right)+o(1) \\
= & \frac{-4}{n-2} \int_{\mathbb{R}^{n}} \delta_{\left(0, \alpha_{n}\right)}^{2 n /(n-2)}-\int_{\mathbb{R}^{n} \backslash B\left(0, \lambda_{i}^{\varepsilon} d_{i}^{\prime}\right)} \delta_{\left(0, \alpha_{n}\right)}^{2 n /(n-2)} \\
& +\frac{n+2}{n-2} \int_{\mathbb{R}^{n} \backslash B(0, R)} \delta_{\left(0, \alpha_{n}\right)}^{2 n /(n-2)}+o(1) .
\end{aligned}
$$

Since $B\left(0, \lambda_{i}^{\varepsilon} d_{i}^{\prime}\right) \rightarrow \mathbb{R}^{n}$, we deduce that

$$
q\left(P_{\varepsilon} \delta_{i}\right) \leq \frac{-4}{n-2} S^{n / 2}+o(1),
$$

where $S$ is the Sobolev constant defined by (2.5).

Clearly, (3.6, ,.., 3.8) give (3.5) and therefore our result follows.

Proof of Theorem 17 Arguing by contradiction, we assume that either the energy is uniformly bounded $\left(H_{1}\right)$, or the Morse index is uniformly bounded $\left(H_{2}\right)$. Using the results of Section 2, we start a blow up process, which enables us to gain at each step at least a fixed amount of energy, and at least 1 in the Morse index. Namely at the $k$ th step, we have constructed $k$ points $\left(a_{1, \varepsilon}, \ldots, a_{k, \varepsilon}\right)$ with concentrations $\left(\lambda_{1, \varepsilon}, \ldots, \lambda_{k, \varepsilon}\right)$ satisfying, as $\varepsilon \rightarrow 0$,

$$
\begin{aligned}
& \forall i \in\{1, \ldots, k\}, \quad \lambda_{i, \varepsilon} d\left(a_{i, \varepsilon}, \partial A_{\varepsilon}\right) \rightarrow+\infty, \\
& \forall R>0 \quad \int_{B\left(a_{1, \varepsilon}, R / \lambda_{1, \varepsilon}\right)} u_{\varepsilon}^{2 n /(n-2)}(x) d x \rightarrow \int_{B(0, R)} \delta_{\left(0, \alpha_{n}\right)}^{2 n /(n-2)}(x) d x, \\
& \forall i \neq 1 \forall R>0 \quad \int_{B\left(a_{i, \varepsilon}, R / \lambda_{i, \varepsilon}\right)} u_{\varepsilon}^{2 n /(n-2)}(x) d x \rightarrow \int_{B\left(b_{i}, R / \mu_{i}\right)} \delta_{\left(b_{i}, \mu_{i}\right)}^{2 n /(n-2)}(x) d x, \\
& \forall i \neq j \in\{1, \ldots, k\}, \quad\left|a_{i, \varepsilon}-a_{j, \varepsilon}\right| \lambda_{i, \varepsilon} \rightarrow+\infty .
\end{aligned}
$$

Hence we derive that

$$
\int_{A_{\varepsilon}}\left|\nabla u_{\varepsilon}\right|^{2} \geq k S^{n / 2} \quad \text { and } \quad m\left(u_{\varepsilon}\right) \geq k .
$$

Then using Propositions 8 and 11 , we derive that this process does not stop after finitely many steps, contradicting assumption $\left(H_{1}\right)$, respectively $\left(H_{2}\right)$. Our theorem follows. 


\section{A Liouville type theorem}

This section is devoted to proving the Liouville type theorem, Theorem 3 stated in the introduction. The main idea is to use the spectral information to gain more integrability of the solution, and this is the content of the next two lemmas.

Lemma 12. Let $u$ be a positive bounded solution of (I). Then

$$
\int_{\Omega} u^{2 n /(n-2)}(x) d x<+\infty .
$$

Proof. Without loss of generality, we may translate the origin in such a way that

$$
\Omega=\left\{\left(x^{\prime}, x_{n}\right) \in \mathbb{R}^{n-1} \times \mathbb{R}: 0<x_{n}<k\right\} \quad(k \text { is a fixed real }) .
$$

We denote by $q$ the quadratic form associated to the linearized operator $-\Delta-\frac{n+2}{n-2} u^{4 /(n-2)}$ defined on $H_{0}^{1}(\Omega) \cap H^{2}(\Omega)$. For $h \in H_{0}^{1}(\Omega) \cap H^{2}(\Omega)$, we have

$$
q(h)=\int_{\Omega}|\nabla h|^{2}-\frac{n+2}{n-2} \int_{\Omega} u^{4 /(n-2)} h^{2} .
$$

Let $d_{0}>0$, and for $R>2 d_{0}$, set

$$
\Omega_{R}=\left\{\left(x^{\prime}, x_{n}\right) \in \mathbb{R}^{n-1} \times \mathbb{R}:\left|x^{\prime}\right|<R, 0<x_{n}<R\right\} .
$$

Now we introduce the function

$$
\varphi_{d_{0}, R}(x)= \begin{cases}0 & \text { if } r \leq d_{0} \\ \frac{r-d_{0}}{d_{0}} & \text { if } d_{0} \leq r \leq 2 d_{0}, \\ 1 & \text { if } 2 d_{0} \leq r \leq R \\ \frac{2 R-r}{R} & \text { if } R \leq r \leq 2 R, \\ 0 & \text { if } r \geq 2 R,\end{cases}
$$

where $r=\left|x^{\prime}\right|$. We distinguish two cases:

Case 1: $\forall R>2 d_{0} \forall \alpha \in(0,1) q\left(\varphi_{d_{0}, R} u^{1+\alpha}\right) \geq 0$.

Case 2: $\exists R_{1}>2 d_{0} \exists \alpha_{1} \in(0,1) \quad q\left(\varphi_{d_{0}, R_{1}} u^{1+\alpha_{1}}\right)<0$.

In Case 1, expanding $q\left(\varphi_{d_{0}, R} u^{1+\alpha}\right)$ and letting $\alpha$ tend to zero, we obtain

$$
\int_{\Omega_{2 R}}|\nabla u|^{2} \varphi_{d_{0}, R}^{2}-\frac{n+2}{n-2} \int_{\Omega_{2 R}} u^{2 n /(n-2)} \varphi_{d_{0}, R}^{2} \geq \int_{\Omega_{2 R}} u^{2}\left(\Delta \varphi_{d_{0}, R}\right) \varphi_{d_{0}, R}
$$

Now, multiplying the equation $-\Delta u=u^{(n+2) /(n-2)}$ by $u^{1+\alpha} \varphi_{d_{0}, R}^{2}$ and integrating by parts on $\Omega_{2 R}$ and letting $\alpha$ tend to zero, we find that

$$
\int_{\Omega_{2 R}}|\nabla u|^{2} \varphi_{d_{0}, R}^{2}-\int_{\Omega_{2 R}} u^{2 n /(n-2)} \varphi_{d_{0}, R}^{2}=\frac{1}{2} \int_{\Omega_{2 R}} u^{2} \Delta\left(\varphi_{d_{0}, R}^{2}\right)
$$


From (4.1) and 4.2), we derive that

$$
\frac{4}{n-2} \int_{\Omega_{2 R}} u^{2 n /(n-2)} \varphi_{d_{0}, R}^{2} \leq \int_{\Omega_{2 R}} u^{2}\left(\frac{1}{2} \Delta\left(\varphi_{d_{0}, R}^{2}\right)-\Delta \varphi_{d_{0}, R} \cdot \varphi_{d_{0}, R}\right) .
$$

Since

we find that

$$
\Delta\left(\varphi_{d_{0}, R}^{2}\right)=2 \varphi_{d_{0}, R} \Delta \varphi_{d_{0}, R}+2\left|\nabla \varphi_{d_{0}, R}\right|^{2}
$$

$$
\frac{4}{n-2} \int_{\Omega_{2 R}} u^{2 n /(n-2)} \varphi_{d_{0}, R}^{2} \leq \int_{\Omega_{2 R}} u^{2}\left|\nabla \varphi_{d_{0}, R}\right|^{2} .
$$

Thus

$$
\frac{4}{n-2} \int_{2 d_{0}<r<R} u^{2 n /(n-2)} \leq \int_{\Omega_{2 R}} u^{2}\left|\nabla \varphi_{d_{0}, R}\right|^{2} .
$$

We now observe that

$$
\begin{array}{ll}
\frac{\partial \varphi_{d_{0}, R}(x)}{\partial x_{i}}=-\frac{x_{i}}{r R} & \text { for } R \leq r \leq 2 R, 1 \leq i \leq n-1, \\
\frac{\partial \varphi_{d_{0}, R}(x)}{\partial x_{i}}=\frac{x_{i}}{r d_{0}} & \text { for } d_{0} \leq r \leq 2 d_{0}, 1 \leq i \leq n-1,
\end{array}
$$

and therefore

$$
\begin{array}{ll}
\left|\nabla \varphi_{d_{0}, R}(x)\right|^{2}=\frac{1}{R^{2}} & \text { for } R \leq r \leq 2 R, \\
\left|\nabla \varphi_{d_{0}, R}(x)\right|^{2}=\frac{1}{d_{0}^{2}} & \text { for } d_{0} \leq r \leq 2 d_{0} .
\end{array}
$$

Thus

$$
\int_{\Omega_{R}} u^{2 n /(n-2)} \leq \frac{1}{R^{2}} \int_{\Omega_{2 R}} u^{2}+c\left(d_{0}\right),
$$

where $c\left(d_{0}\right)$ is a positive constant depending only on $d_{0}$ and $n$. Using Hölder's inequality, we find that

$$
\int_{\Omega_{R}} u^{2 n /(n-2)} \leq \frac{c}{R^{2}}\left(\int_{\Omega_{2 R}} u^{2 n /(n-2)}\right)^{(n-2) / n} R^{2(n-1) / n}+c\left(d_{0}\right) .
$$

That is,

$$
\int_{\Omega_{R}} u^{2 n /(n-2)} \leq \frac{c}{R^{2 / n}}\left(\int_{\Omega_{2 R}} u^{2 n /(n-2)}\right)^{(n-2) / n}+c\left(d_{0}\right) .
$$

Since $0 \leq u \leq c$ on $\Omega$, we deduce that

$$
\int_{\Omega_{2 R}} u^{2 n /(n-2)} \leq c R^{n-1} .
$$

Therefore by [4.3, we have

$$
\int_{\Omega_{R}} u^{2 n /(n-2)} \leq c R^{n-3}+c\left(d_{0}\right) .
$$


We insert this bound in 4.3 and iterate this argument to obtain

$$
\int_{\Omega_{R}} u^{2 n /(n-2)} \leq c R^{\alpha_{p}}+c^{\prime}\left(d_{0}\right)
$$

with $\alpha_{0}=n-3, \alpha_{p+1}=\frac{n-2}{n} \alpha_{p}-\frac{2}{n}$ and $c^{\prime}\left(d_{0}\right)$ a positive constant depending only on $d_{0}$ and $n$. It is easy to see that $\alpha_{p}$ converges to -1 when $p$ tends to $\infty$. Taking $p_{0}$ such that $\alpha_{p_{0}}<0$, we then derive

$$
\int_{\Omega} u^{2 n /(n-2)}<\infty
$$

in the first case.

In Case 2, the Morse index of $u$ is at least 1. Consider $d_{1}>2 R_{1}$. Then either $q\left(\varphi_{d_{1}, R} u^{1+\alpha}\right) \geq 0$ for all $R>2 d_{1}$ and all $\alpha \in(0,1)$ (as in the first case we then prove that $\left.\int_{\Omega} u^{2 n /(n-2)}<\infty\right)$ or there exist $R_{2}>2 d_{1}, \alpha_{2} \in(0,1)$ such that $q\left(\varphi_{d_{1}, R_{2}} u^{1+\alpha_{2}}\right)<0$. Since $d_{1}>2 R_{1}$, the supports of $\varphi_{d_{0}, R_{1}}$ and $\varphi_{d_{1}, R_{2}}$ are disjoint and therefore the Morse index of $u$ is larger than or equal to 2 . We again iterate this argument. Since $m(u)<\infty$, there exists $d>0$ such that

$$
q\left(\varphi_{d, R} u^{1+\alpha}\right) \geq 0, \quad \forall \alpha \in(0,1), \forall R>2 d .
$$

Then, as in the first case, we prove that 4.4 implies $\int_{\Omega} u^{2 n /(n-2)}<\infty$, and the lemma follows.

Lemma 13. Let u be a positive bounded solution of (I). Then

$$
\int_{\Omega}|\nabla u|^{2} d x<\infty
$$

Proof. For $\varepsilon>0$ small let $h=h_{\varepsilon} \in C_{c}^{1}(\Omega)$ be a cut-off function such that

$$
0 \leq h \leq 1, \quad h(x)=\left\{\begin{array}{ll}
1 & \text { if } x \in \Omega_{1 / \varepsilon}, \\
0 & \text { in } \Omega \backslash \Omega_{2 / \varepsilon} ;
\end{array} \quad|\nabla h| \leq 2 \varepsilon \quad \text { in } \Omega_{2 / \varepsilon} \backslash \Omega_{1 / \varepsilon},\right.
$$

where, for $l>0, \Omega_{l}$ is the subset of $\Omega$ defined by

$$
\Omega_{l}=\left\{\left(x^{\prime}, x_{n}\right) \in \mathbb{R}^{n-1} \times \mathbb{R}:\left|x^{\prime}\right|<l \text { and } 0<x_{n}<k\right\} .
$$

(We recall that after translation we may suppose that $\Omega=\left\{\left(x^{\prime}, x_{n}\right) \in \mathbb{R}^{n-1} \times \mathbb{R}: 0<\right.$ $\left.x_{n}<k\right\}$, where $k$ is a fixed real.) We then test the equation

$$
-\Delta u=u^{(n+2) /(n-2)}
$$

with the function $\varphi=\varphi_{\varepsilon}=u h^{2}$ to obtain estimates for the function $\psi=\psi_{\varepsilon}=u h$. Observe that

$$
\begin{aligned}
\nabla \varphi & =h^{2} \nabla u+2 u h \nabla h, \\
\nabla u \nabla \varphi & =h^{2}|\nabla u|^{2}+2 u h \nabla h \cdot \nabla u, \\
|\nabla \psi|^{2} & =h^{2}|\nabla u|^{2}+2 u h \nabla u \nabla h+u^{2}|\nabla h|^{2} \\
& =\nabla u \nabla \varphi+u^{2}|\nabla h|^{2} .
\end{aligned}
$$


Thus

$$
\int_{\Omega}|\nabla \psi|^{2}=\int_{\Omega} \nabla u \nabla \varphi+\int_{\Omega} u^{2}|\nabla h|^{2}=\int_{\Omega} u^{2 n /(n-2)} h^{2}+\int_{\Omega} u^{2}|\nabla h|^{2} .
$$

Using Lemma 12 Hölder's inequality and the fact that $|\nabla h| \leq 2 \varepsilon$ in $\Omega_{2 / \varepsilon} \backslash \Omega_{1 / \varepsilon}$, we derive that

$$
\int_{\Omega}|\nabla \psi|^{2} \leq \int_{\Omega} u^{2 n /(n-2)} h^{2}+c .
$$

Letting $\varepsilon$ tend to zero, we derive our lemma.

Proof of Theorem 3 Using Lemmas 12 and 13 and the Pohozaev identity, we derive that $u$ vanishes identically (see e.g. [25, Theorem 1.3, p. 156]).

\section{Appendix: blow up analysis}

In this appendix, we give the definitions, and recall basic properties of isolated and isolated simple blow up points, which were first introduced by R. Schoen [21], [22], [23] and extensively studied by Y.Y. Li [17].

Let $\Omega \subseteq \mathbb{R}^{n}$ be a bounded smooth domain. Consider a family $\left(u_{i}\right)$ of solutions to the following equation:

$$
-\Delta u=u^{n+2 / n-2}, \quad u>0 \quad \text { in } \Omega .
$$

The aim of the blow up analysis is to describe the behavior of $u_{i}$ as $i$ tends to infinity. It follows from standard elliptic regularity that if $\left\{u_{i}\right\}_{i}$ remains bounded in $L_{\mathrm{loc}}^{\infty}(\Omega)$, then for any $\alpha \in(0,1), u_{i} \rightarrow u$ in $C_{\text {loc }}^{2, \alpha}(\Omega)$ along some subsequence. Otherwise, we say that $\left\{u_{i}\right\}_{i}$ blows up. Let $B_{r}(x)=\left\{y \in \mathbb{R}^{n}:|y-x|<r\right\}$.

Definition 14. Suppose that $\left\{u_{i}\right\}_{i}$ satisfy (5.1). A point $\bar{y} \in \Omega$ is called a blow up point for $\left\{u_{i}\right\}_{i}$ if there exist $y_{i} \rightarrow \bar{y}$ such that $u_{i}\left(y_{i}\right) \rightarrow+\infty$.

In what follows, if $\bar{y}$ is a blow up point for $\left\{u_{i}\right\}_{i}$, writing $y_{i} \rightarrow \bar{y}$ we mean that $y_{i} \rightarrow \bar{y}$ and $u_{i}\left(y_{i}\right) \rightarrow+\infty$ as $i \rightarrow+\infty$.

Definition 15. Assume that $y_{i} \rightarrow \bar{y}$ is a blow up point for $\left\{u_{i}\right\}_{i}$. The point $\bar{y} \in \Omega$ is called an isolated blow up point if there exist $\bar{r} \in(0, d(\bar{y}, \partial \Omega))$ and $\bar{C}>0$ such that

$$
u_{i}(y) \leq \bar{C}\left|y-y_{i}\right|^{-(n-2) / 2} \quad \text { for all } y \in B_{\bar{r}}\left(y_{i}\right) \cap \Omega .
$$

Isolated blow up enjoys nice properties, such as a Harnack inequality around singular points:

Lemma 16 ([17]). Let $u_{i}$ satisfy (5.1] and $y_{i} \rightarrow \bar{y} \in \Omega$ be an isolated blow up point of $\left\{u_{i}\right\}_{i}$. Then for any $0<r<\bar{r}$, we have

$$
\max _{B_{2 r}\left(y_{i}\right) \backslash B_{r / 2}\left(y_{i}\right)} u_{i} \leq C_{3} \min _{B_{2 r}\left(y_{i}\right) \backslash B_{r / 2}\left(y_{i}\right)} u_{i},
$$

where $C_{3}$ is some positive constant independent of $i$ and $r$. 
The property of being isolated prevents accumulation of blow up points, but it does not prevent superposition of bubbles over bubbles. For this we need the notion of isolated simple blow up. Let $y_{i} \rightarrow \bar{y}$ be an isolated blow up point for $\left\{u_{i}\right\}_{i}$. We define (here $\left|\partial B_{r}\right|$ is the $n-1$-dimensional volume of $\partial B_{r}$ )

$$
\bar{u}_{i}(r)=\frac{1}{\left|\partial B_{r}\right|} \int_{\partial B_{r}\left(y_{i}\right)} u_{i}, \quad r \in\left(0, d\left(y_{i}, \partial \Omega\right)\right),
$$

and

$$
\hat{u}_{i}(r)=r^{(n-2) / 2} \bar{u}_{i}(r), \quad r \in\left(0, d\left(y_{i}, \partial \Omega\right)\right) .
$$

Definition 17. An isolated blow up point $\bar{y} \in \Omega$ for $\left\{u_{i}\right\}_{i}$ is called an isolated simple blow up point if there exists some $\varrho \in(0, \bar{r})$, independent of $i$, such that $\hat{u}_{i}(r)$ has precisely one critical point in $(0, \varrho)$ for large $i$.

The property of being an isolated simple blow up point means that in a ball of fixed radius around the blow up point, the solution is upper bounded and lower bounded by a constant times the bubble. In the following lemma, we give a quantitative statement of this fact.

Proposition 18 ([17]). Assume that $\left\{u_{i}\right\}_{i}$ satisfies $\left[5.1\right.$ with $\Omega=B_{2}$, and let $y_{i} \rightarrow$ $\bar{y} \in \Omega$ be an isolated simple blow up point for $\left\{u_{i}\right\}_{i}$, which for some positive constant $M$ satisfies

$$
\left|y-y_{i}\right|^{(n-2) / 2} u_{i}(y) \leq M, \quad \forall y \in B_{2} .
$$

Then there exists some positive constant $C=C(n, M, \varrho)$ ( $\varrho$ being given in the definition of isolated simple blow up point) such that for $0<\left|y-y_{i}\right| \leq 1$,

$$
C^{-1} u_{i}\left(y_{i}\right)^{-1}\left|y-y_{i}\right|^{2-n} \leq u_{i}(y) \leq C u_{i}\left(y_{i}\right)^{-1}\left|y-y_{i}\right|^{2-n} .
$$

The main result of the blow up analysis of Yamabe type equations on locally conformally flat manifolds is that all isolated blow up points are actually isolated simple blow up points. This is recalled in

Proposition 19 ([17]). Assume that $\left\{u_{i}\right\}_{i}$ satisfies equation (5.1) on $\Omega=B_{2} \subset \mathbb{R}^{n}$ $(n \geq 3)$ and let $\bar{y}$ be an isolated blow up point for $\left\{u_{i}\right\}_{i}$. Then $\bar{y}$ is an isolated simple blow up point.

Acknowledgements. Part of this work was done while the first and the second author were visiting the Mathematics Department of the University of Rome "La Sapienza". They would like to thank the Mathematics Department for its warm hospitality.

\section{References}

[1] Aubin, T., Bahri, A.: Une remarque sur l'indice et la norme infinie des solutions d'équations elliptiques surlinéaires. Ricerche Mat. 48, suppl., 117-128 (1999) Zbl 0932.35083 MR 1765680 
[2] Bahri, A.: Topological results on a certain class of functionals and applications. J. Funct. Anal. 41, 397-427 (1981) Zbl 0499.35050 MR 0619960

[3] Bahri, A.: Critical Points at Infinity in Some Variational Problems. Pitman Res. Notes Math. Ser. 182, Longman Sci. Tech., Harlow (1989) Zbl 0676.58021 MR 1019828

[4] Bahri, A., Lions, P. L.: Morse index of some min-max critical points, I: Application to multiplicity results. Comm. Pure Appl. Math. 41, 1027-1037 (1988) Zbl 0645.58013 MR 0968487

[5] Bahri, A., Lions, P. L.: Solutions of superlinear elliptic equations and their Morse indices. Comm. Pure Appl. Math. 45, 1205-1215 (1992) Zbl 0801.35026 MR 1177482

[6] Ben Ayed, M., El Mehdi, K., Hammami, M.: A nonexistence result for Yamabe type problems on thin annuli. Ann. Inst. H. Poincaré Anal. Non Linéaire 19, 715-744 (2002) Zbl pre01801807 MR 1922475

[7] Ben Ayed, M., El Mehdi, K., Hammami, M., Ould Ahmedou, M.: On a Yamabe type problem on three dimensional thin annulus. Preprint, The Abdus Salam ICTP, IC/2004/38, Trieste

[8] Bénichou, A., Pomet, J.-B.: The index of the radial solution of some elliptic P.D.E. Nonlinear Anal. 14, 991-997 (1990) Zbl 0717.35027 MR 1058418

[9] Caffarelli, L., Gidas, B., Spruck, J.: Asymptotic symmetry and local behavior of semilinear elliptic equations with critical Sobolev growth. Comm. Pure Appl. Math. 42, 271-297 (1989) Zbl 0702.35085 MR 0982351

[10] De Figueiredo, D., Yang, J.: On a semilinear elliptic problem without (PS) condition. J. Differential Equations 187, 412-428 (2003) Zbl pre01884229 MR 1949448

[11] El Mehdi, K., Pacella, F.: Morse index and blow-up points of solutions of some nonlinear problems. Rend. Accad. Naz. Lincei 13, 101-105 (2002) MR 1949483

[12] Gidas, B., Spruck, J.: A priori bounds for positive solutions of nonlinear elliptic equations. Comm. Partial Differential Equations 6, 883-901 (1981) Zbl 0462.35041 MR 0619749

[13] Gilbarg, D., Trudinger, N.S.: Elliptic Partial Differential Equations of Second Order. 2nd ed., Grundlehren Math. Wiss. 224, Springer, Berlin (1983) Zbl 0562.35001 MR 0737190

[14] Harrabi, A., Rebhi, S., Selmi, A.: Solutions of superlinear elliptic equations and their Morse indices. Duke Math. J. 94, I: 141-157, II: 159-179 (1998) Zbl 0952.35042 MR 1635912

[15] Kellogg, O. D.: Foundations of Potential Theory, Dover (1953) Zbl 0053.07301

[16] Lazer, M., Solimini, S.: Nontrivial solutions of operator equations and Morse indices of critical points of min-max type. Nonlinear Anal. 10, 411-413 (1986) Zbl 0619.58011 MR 0836676

[17] Li, Y. Y.: Prescribing scalar curvature on $S^{n}$ and related topics, Part I. J. Differential Equations 120, 319-410 (1995) Zbl 0827.53039 MR 1347349

[18] Pacella, F.: Symmetry results for solutions of semilinear elliptic equations with convex nonlinearities. J. Funct. Anal. 192, 271-282 (2002) Zbl 1014.35032 MR 1918496

[19] Ramos, M., Terracini, S., Troestler, C.: Superlinear indefinite elliptic problems and Pohozaev identity. J. Funct. Anal. 159, 596-628 (1998) Zbl 0937.35060 MR 1658097

[20] Rey, O.: The role of Green's function in a nonlinear elliptic equation involving the critical Sobolev exponent. J. Funct. Anal. 89, 1-52 (1990) Zbl 0786.35059 MR 1040954

[21] Schoen, R.: Variational theory for the total scalar curvature functional for Riemannian metrics and related topics. In: Topics in Calculus of Variations (Montecatini Terme, 1987), Lecture Notes in Math. 1365, Springer, Berlin, 120-154 (1989) Zbl 0702.49038 MR 0994021

[22] Schoen, R.: Courses at Stanford University (1988) and New York University (1989), unpublished

[23] Schoen, R.: On the number of solutions of constant scalar curvature metrics in a conformal class. In: Differential Geometry: A Symposium in Honor of Manfredo Do Carmo (H. B. Lawson and K. Tenenblat, eds.), Wiley, 311-320 (1991) 
[24] Solimini, S.: Morse index estimates in min-max theorems. Manuscripta Math. 63, 421-453 (1989) Zbl 0685.58010 MR 0991264

[25] Struwe, M.: Variational Methods. Applications to Nonlinear Partial Differential Equations and Hamiltonian systems. Springer, Berlin (1990) Zbl 0746.49010 MR 1078018

[26] Yang, X. F.: The Morse critical groups and the min-max theorems. Chinese Ann. Math. Ser. B 12, 202-206 (1991) Zbl 0731.58017 MR 1112255

[27] Yang, X. F.: Nodal sets and Morse indices of solutions of super-linear elliptic PDEs. J. Funct. Anal. 160, 223-253 (1998) Zbl 0919.35049 MR 1658692 